\title{
TITANIUM ALLOYS AND PROCESSING FOR HIGH SPEED AIRCRAFT
}

\author{
William D. Brewer, R. Keith Bird, and Terryl A. Wallace \\ NASA Langley Research Center \\ Hampton Virginia 23681-0001
}

\begin{abstract}
Commercially available titanium alloys as well as emerging titanium alloys with limited or no production experience are being considered for a variety of applications to high speed commercial aircraft structures. A number of government and industry programs are underway to improve the performance of promising alloys by chemistry and/or processing modifications and to identify appropriate alloys and processes for specific aircraft structural applications. This paper discusses some of the results on the effects of heat treatment, service temperatures from $54^{\circ} \mathrm{C}$ to $+177^{\circ} \mathrm{C}$, and selected processing on the mechanical properties of several candidate beta and alpha-beta titanium alloys. Included are beta alloys Timetal 21S, LCB, Beta C, Beta CEZ, and Ti-10-2-3 and alpha-beta alloys Ti-62222, Ti-6242S, Timetal 550, Ti-62S, SP-700, and Corona-X. The emphasis is on properties of rolled sheet product form and on the superplastic properties and processing of the materials.
\end{abstract}

\section{Introduction}

As economic pressures increase for commercial transport, the demand for reduced weight and improved performance becomes greater. For some proposed applications and missions such as a supersonic transport operating at speeds above Mach 2, titanium alloys play an increasingly critical role and in fact may be the enabling technology. However, improvements in performance, processibility and cost must be forthcoming in order to realize the potential of an economically viable supersonic transport. Each class of titanium alloy has its advantages and disadvantages, and the key is to develop an understanding of the behavior of the alloys and to match particular alloys and processes to the appropriate application to maximize structural and operational efficiency. Equally important is the ability to maintain fabrication practice that does not severely degrade material properties and can be accomplished at reasonable cost. This paper reviews some of the NASA sponsored research to develop titanium alloys and associated fabrication practice for application to airframe structures operating at speeds above Mach 2.0. The emphasis is on significantly improving the mechanical properties of titanium alloys over those of industry standards (Ti-6Al-4V, e.g.) while maintaining acceptable fabricability and long term stability in projected service environments.

\section{Material Requirements}

Figure 1 shows schematically the operational/environmental requirements for several classes of aircraft operating in various mission environments. Current commercial transports are designed for long service life $(\sim 60,000 \mathrm{hrs})$ but operate in the relative benign lower temperature range whereas the high performance aircraft operate in the high speed, and hence high temperature, regime but for relatively short times. Next generation high speed transports will be required to operate in both the elevated temperature and long life regimes. Among the key technology needs 
for some proposed high speed transports is the development of lightweight materials capable of operating at temperatures from $-54{ }^{\circ} \mathrm{C}$ to $177{ }^{\circ} \mathrm{C}$ for times greater than $60,000 \mathrm{hrs}$. Because aluminum alloys are generally not capable of operating under most of these conditions, and high temperature polymeric composites are in a relatively early stage of development, titanium is a natural selection for potential applications to these kinds of environments.

\section{Candidate Alloys}

Titanium alloy development is underway primarily to optimize combinations of strength, toughness, and stiffness for selected applications to airframe structures while maintaining other critical properties such as fatigue and crack growth resistance at acceptable levels. Table 1 shows the alloys under consideration and lists some of the rationale for including each one in the list. The alloys range from mature (Ti-6242S, Timetal 550, Ti-10-2-3, Beta-C, e.g.), to relatively new (Ti-62222, SP 700, Ti-62S, Beta CEZ, Timetal $21 \mathrm{~S}$ ) to limited experience or experimental (LCB, Corona $\mathrm{X}$ ). Ti-6242S is a mature alloy developed for high tensile and creep strength and high temperature stability. However, in the current programs, the alloy is used in the $\mathrm{RX} 2$ condition, a heat treat process developed by researchers at Rockwell International that yields a very high modulus (> $130 \mathrm{GPa}$ ) while maintaining relatively high strength and toughness. Ti-62222 saw limited development until it was selected as baseline for the F-22 fighter aircraft. That application is primarily for thick section components and performance in sheet product form is largely unknown. Likewise, Ti-10-2-3, Beta CEZ, and Beta-C were developed primarily for uses in heavy section parts. Corona $X$ is an experimental alloy derived from Corona 5 that was developed by Rockwell and the Navy as a moderate strength, high toughness alloy (Ref. 1). In thick section, it shows improved strength and toughness over Corona 5. SP 700 is a relatively new high formability alloy developed by NKK corporation. It has very good superplastic forming characteristics at temperatures below $800^{\circ} \mathrm{C}$ and has relatively high toughness. Timetal-21S is a high strength alloy developed for improved oxidation resistance. It has very good resistance to hydraulic fluids such as Skydrol. Timetal LCB was developed as a high strength, low cost alloy and has been marketed primarily for coil spring applications. All the alloys are being evaluated in sheet product form. Data given herein are from sheet product ranging from $1 \mathrm{~mm}$ to $2 \mathrm{~mm}$ thick.

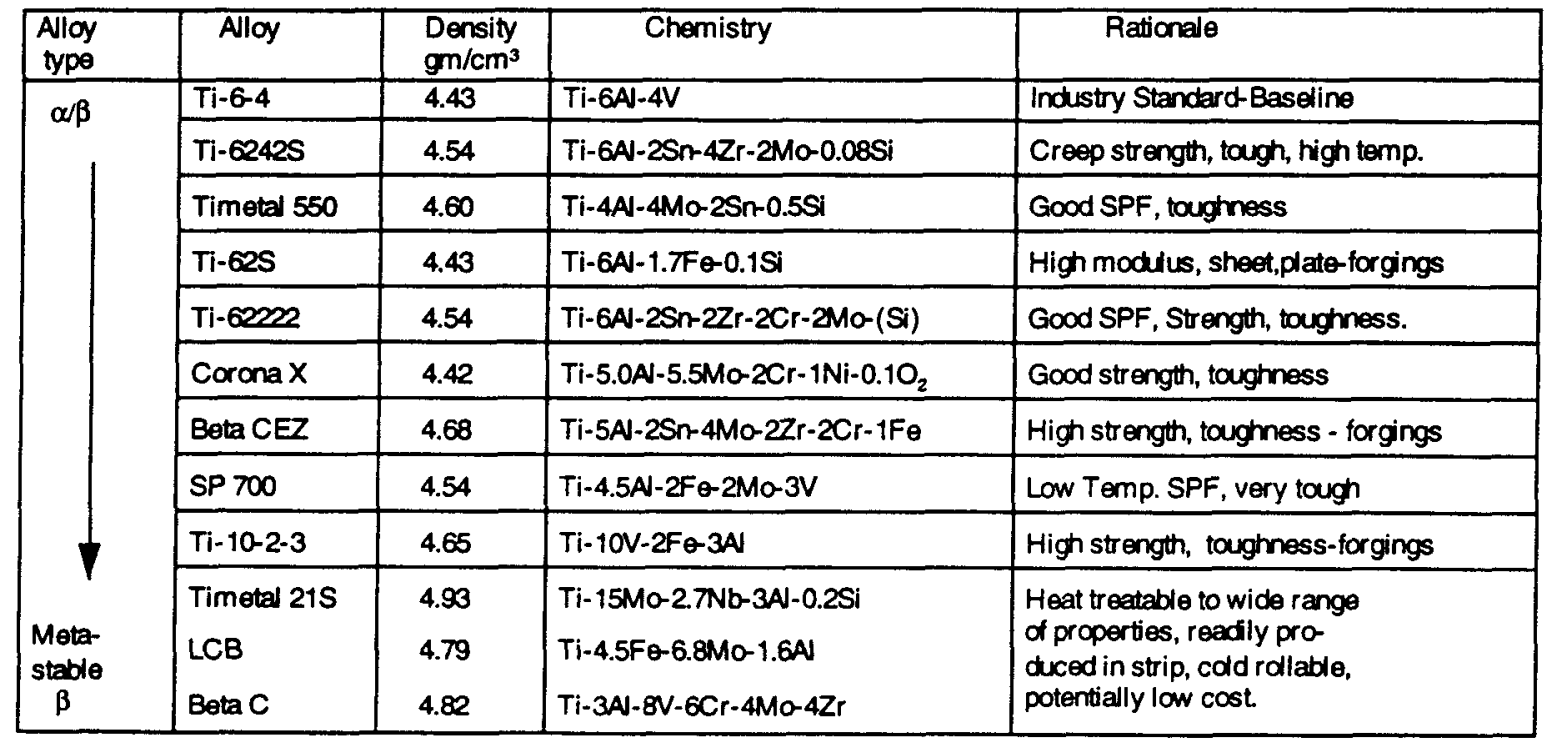

Table 1.- Candidate Advanced Titanium Alloys for High Speed Aircraft Structures

\section{Alloy Testing \& Performance}

Tensile and toughness testing has been conducted on most of the alloys listed in Table 1 at -54 ${ }^{\circ} \mathrm{C}$, room temperature, and $177^{\circ} \mathrm{C}$. For screening purposes, most of the toughness testing has utilized the J-integral versus crack growth resistance curve (J-R curve) method (Refs. 2, 3, 4). This method uses small compact tension specimens and can give a good indication of the toughness of sheet material without having to use large test samples that consume valuable and 
scarce materials. When the most promising alloys and treatments have been identified, wide sheet testing is performed to obtain more definitive data.

Figure 2 shows room temperature crack growth resistance curves for Ti-62222 aged for 8 hrs at four different aging temperatures. All the samples were solution treated at $918^{\circ} \mathrm{C}$ for 1 hour. Earlier data showed that variations in solution temperatures from about $885^{\circ} \mathrm{C}$ to about $930^{\circ} \mathrm{C}$ do not have a significant effect on tensile properties. Solutionizing at $970^{\circ} \mathrm{C}$ however caused a large drop in strength and ductility. In general, the higher the curve on the plot in Figure 2, the better the crack growth resistance. The $\mathrm{K}_{\mathrm{Jl}}$ fracture toughness values calculated from these curves and the corresponding yield strengths are tabulated in the figure. Both the strength and toughness are seen to be maximum at the $540{ }^{\circ} \mathrm{C}$ age. Work is underway to determine the fundamental causes for the loss in toughness for this alloy at the higher aging temperatures.

Room temperature crack growth resistance curves for several of the other alloys under consideration are given in Figure 3, along with the Ti-62222 $540{ }^{\circ} \mathrm{C}$ age curve from Figure 2 . The heat treatments shown are those that gave some of the best results for the particular alloys. Typically, the high strength conditions of Beta-C and Timetal 21S showed the lower toughness. Even when Timetal $21 \mathrm{~S}$ was treated to a lower strength (1055 MPa) comparable to that of Ti$6242 \mathrm{~S}(1048 \mathrm{MPa})$ and lower than that of Ti-62222 (1207 MPa), the toughness was still considerably lower than that for either of the alpha/beta alloys. Likewise for Beta- $\mathrm{C}$, the lower strength age resulted in strengths and toughness less than those of Ti-62222. The Ti-10-2-3 alloy had the highest toughness, but the lowest strength. Of the alloys shown in Figure 3, the Ti-6-2222 with the $540^{\circ} \mathrm{C}$ age exhibited the best combination of strength and toughness.

Figure 4 shows the fracture toughness versus yield strength of selected alloys at $-54^{\circ} \mathrm{C}$, room temperature, and $177^{\circ} \mathrm{C}$ (Timetal $21 \mathrm{~S}$ only). Also shown for reference is a band of room temperature data for Timetal $21 \mathrm{~S}$ representing many heat treat conditions (Ref. 5). Even though the temperature range is somewhat modest relative to conventional wisdom regarding titanium performance, the temperature does have a significant effect on the properties of all the alloys. At $-54^{\circ} \mathrm{C}$ the strengths of the alloys increase from about $13 \%$ (Timetal $21 \mathrm{~S}$ ) to about $22 \%$ (Ti-102-3) while the toughness drops from as little as 9\% (Ti-6242S) to as much as $40 \%$ (Timetal $21 S$ ). As indicated by the Timetal $21 S$ data, the strength-toughness trend is expected to continue to the higher temperatures where the strength decreases and toughness increases significantly relative to room temperature properties. Although the properties of all the alloys are affected by the temperature, the strength-toughness trends seem to follow the overall trend for the room temperature Timetal $21 \mathrm{~S}$ properties. This seems to suggest that, for any particular alloy within this temperature range, a given strength level will yield a given toughness regardless of the temperature. Again, the alpha-beta alloys lie at the upper level of data and seem to be a little less sensitive to temperature.

\section{Fabrication Technology}

Titanium fabrication technology development is focused on sandwich structure, including superplastic forming/diffusion bonded truss core (roll seam welded and laser welded), and metallurgically and adhesively bonded honeycomb core. Other activities include the development of high modulus extrusions, and an assessment of the applicability of more conventional forming methods such as break-, hydro-, and stretch-forming, bending and joggling to the advanced titanium alloys. Small efforts are also underway to assess the potential of warm rolling alpha/beta alloys for lower cost sheet product and reconfigurable tooling to reduce the cost of titanium parts. To date the largest effort has been on superplastic forming (SPF) to determine the SPF characteristics of a number of advanced titanium alloys and to assess their potential for application to sandwich structure. Among the key attributes for a good SPF material are practical SPF temperatures, low flow stress at the forming temperatures and high strain capability. Figure 5 shows the flow stress as a function of SPF temperature for several candidate alloys. The flow stress here is defined as the pressure required to form the material into a particular tool. From the flow stress perspective, the clear choices seem to be the alpha/beta alloys Timetal-550, Ti-62222, and SP-700 with the Ti-62222 showing the lowest flow stresses and SP-700 with relatively low stresses at the lowest processing temperatures. 
The strain capacity of various alloys at representative SPF temperatures is shown in Figure 6. The numbers at the top of the bars indicate the strain at failure. The symbol $\left(^{*}\right)$ at the top of a bar indicates that the test was stopped at $2000 \%$ strain with no testpiece failure. The Ti-62222, Timetal 550, and SP-700 all produced elongations more that $2000 \%$ at the appropriate temperatures. Because of this behavior and the relatively low flow stresses at these temperatures (Figure 5) these alloys are considered prime candidates for superplastic forming applications. As can be seen in the figure, the other alloys can be made to behave superplastically, but it is a much more difficult operation and the elongations to failure are much lower.

Most of the alloys in Figures $5 \& 6$ have been used to fabricate multi-sheet truss core sandwich structure using superplastic forming processing. These structural concepts are candidates for a variety of applications on high speed aircraft. (Ref. 6). Two basic processes have been used to fabricate truss core test panels: roll seam welding/superplastic forming/diffusion bonding (RSW/SPF/DB), developed primarily at McDonnell Douglas Aerospace in Long Beach, CA, and laser welding/superplastic forming/diffusion bonding (LW/SPF/DB) being developed at Boeing Defense and Space Group in Seattle, WA. Schematic diagrams of the RSW/SPF/DB process for 4 sheet rectangular cell and 5 sheet crossweb structure are given in Figure 7. Generally, the internal sheets are roll seam welded in the appropriate pattern. Outer face sheets are added and the periphery of the titanium stack is welded. The face sheets are expanded into a tool, the inner sheets are expanded to the face sheets and to form the webs, and the entire pack is then put through an appropriate diffusion bonding cycle, the conditions of which depend upon the alloys being used. The 4-sheet structure is a very efficient structure, but for some applications, concern has been expressed about the possible lack of damage tolerance in that design. For that reason, McDonnell Douglas developed designs and processes using 5 or more sheets. The 5-sheet design has a double face sheet formed on what would be the exterior side of the panel. This configuration effectively has a redundant load path in case of a failure in the outer face sheet. It is however considerably more difficult to fabricate. Figure 8 shows a typical panel fabricated by the 5 sheet process. The RSW/SPF/DB process has been used to fabricate 4 and 5 sheet panels up to about $1 \mathrm{~m} \mathrm{x} 1.5 \mathrm{~m}$ with several of the advanced alloys and with combinations of alloys in the same panel. The process is being scaled up to larger structure. As expected from their SPF properties, the Timetal 550, Ti-62222 and SP 700 are readily amenable to this process. The SP 700 , however, has shown a tendency for excessive thinning in the areas around the welds causing some concern about the utility of the alloy in this application.

Figure 9 shows the 3 sheet and 4 sheet LW/SPF/DB process. For the 3 sheet process, two titanium sheets are laser welded in the appropriate pattern. The third sheet is then welded on top of the 2-sheet pack with partial penetrating welding. The total pack is placed in a tool and expanded into the sandwich configuration resulting in a triangular cell structure. The 3-sheet process generally does not include additional face sheets and hence a diffusion bonding cycle is not required. The 4-sheet process involves welding 2 sheets in the desired pattern, placing the 2-sheet core pack along with 2 external face sheets in a sealed envelope, superplastically forming the core pack and diffusion bonding to the face sheets to form the final configuration. A typical laser welding operation and 4-sheet rectangular core panel are shown in Figure 9. As with the RSW/SPF/DB process, the LW/SPF/DB process has been successfully used to make high quality panels with several of the advanced alloys.

\section{Summary}

A wide variety of beta and alpha-beta titanium alloys and associated processes are being investigated for potential application to high speed aircraft structures. Thermomechanical processing is being optimized to produce alloys with microstructures that show promise for yielding the required properties. In general, the alpha-beta alloys have shown some of the better combinations of properties with less effects of temperature in the $-54{ }^{\circ} \mathrm{C}$ to $177^{\circ} \mathrm{C}$ range than the beta alloys. Ti-62222 and Timetal 550 have shown high toughness at moderate to high strengths with good fabricability in sheet form. SP 700 has very good toughness and SPF characteristics, but excessive thinning near welds in SPF/DB structure could be an issue. The Ti-6242S with the Rockwell RX2 heat treatment has a very high modulus, comparable that of Ti-62S, but with better strength and toughness. The experimental alloy Corona $X$ has shown properties approaching those for conventional alloys in thick plate but has yet to be evaluated in 
sheet form. The beta alloys Timetal 21S, Beta-C, and LCB can be processed to very high strengths, but the toughness is typically low. Even when heat treated to equivalent strengths as some of the alpha-beta alloys the toughness remains relatively low. Because of high flow stresses the beta alloys are less attractive for SPF applications. Although significant progress has been made in developing chemistries and processing that produce significant gains in material performance over commercially available materials, further progress is required to meet the weight and economic requirements of proposed high speed aircraft.

\section{Acknowledgments}

The authors wish to acknowledge Ray Tisler of McDonnell Douglas Aerospace Co., Long Beach, CA, and Jeff Will of the Boeing Defense and Space Group, Seattle, WA working under contract NAS1-20220 for SPF/DB contributions to this paper.

\section{References}

1. Martin, P.L.: "Toughness-Strength Trend in Alloys Derived from Ti-4.5Al-5Mo-1.5Cr." Proceedings of the Eighth World Conference on Titanium. Birmingham, England 1995. pp 940-947.

2. ASTM E1152-87: "Standard Test Method for Determining J-R Curves", 1992 Annual Book of ASTM Standards. Vol. 03.01, Amer. Soc. of Testing and Materials, 1992, pp 847-857

3. ASTM E466-82: "Standard Test Method for J ${ }_{1 \mathrm{C}}$, A Measure of Fracture Toughness." 1992 Annual Book of ASTM Standards. Vol. 03.01, American Society of Testing and Materials, 1992, pp 732-746.

4. Hertzberg, Richard W. Deformation and Fracture Mechanics of Engineering Materials, New York, John Wiley \& Sons, 1983

5. Bird, R.K.; Wallace, T.A.; and Brewer, W.D.: "Effects of Heat Treatment on Microstructure, Strength, and Toughness of Timetal 21S", Proceedings of the 125th TMS Annual Meeting, 1996

6. Hatakeyama, Jason: "Wing Structures Development for the HSCT." Aerospace Engineering, May 1995, pp 25-29

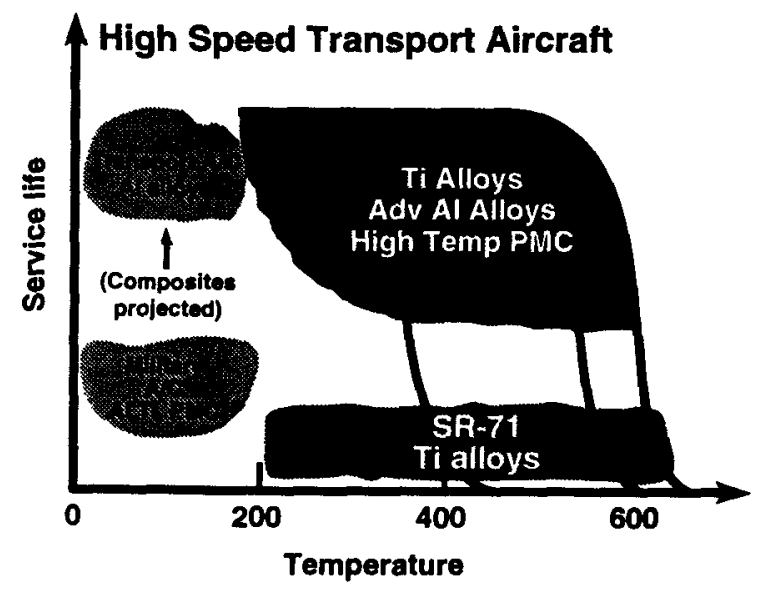

Figure 1. Typical materials and service environments for various aircraft.

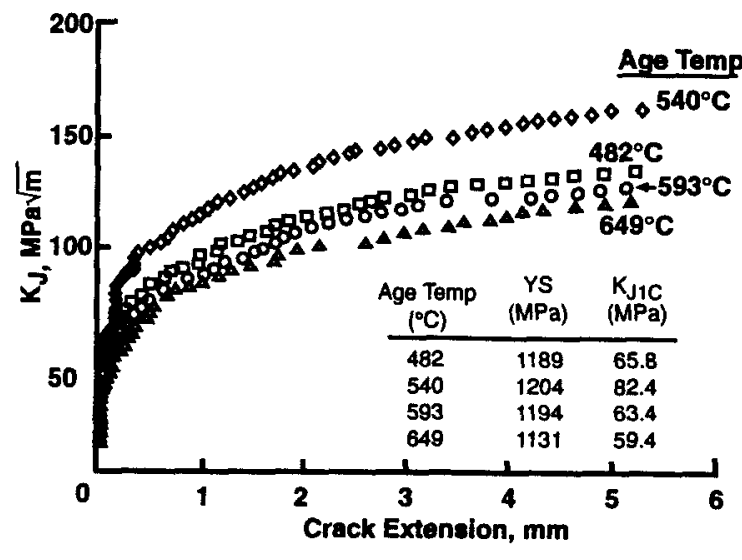

Figure 2. Room temperature crack growth resistance curves for Ti-62222. Four aging conditions. 


\begin{tabular}{|c|c|c|c|c|}
\hline Symbol & Alloy & Gondition & Ys & $\overline{K_{10}}$ \\
\hline & & & MPa & $M P a \sqrt{m}$ \\
\hline 4 & $\pi-2222$ & $540^{\circ} \mathrm{C}$ age & 1207 & 82.3 \\
\hline 0 & $\pi-62428$ & $A \times 2$ & 1040 & 05.4 \\
\hline$\square$ & $7-10-2-3$ & $510^{\circ} \mathrm{Caga}$ & 869 & 86.8 \\
\hline - & Timat. 219 & $620^{\circ} \mathrm{C}$ age & 1055 & 74.6 \\
\hline 0 & ninet 213 & $520^{\circ} \mathrm{C}$ ago & 1448 & 57.5 \\
\hline$\nabla$ & $\beta-C$ & $550^{\circ} \mathrm{C}$ age & 1150 & 65.1 \\
\hline$\nabla$ & $\beta-C$ & $480^{\circ} \mathrm{C}$ age & 1317 & 51.4 \\
\hline
\end{tabular}

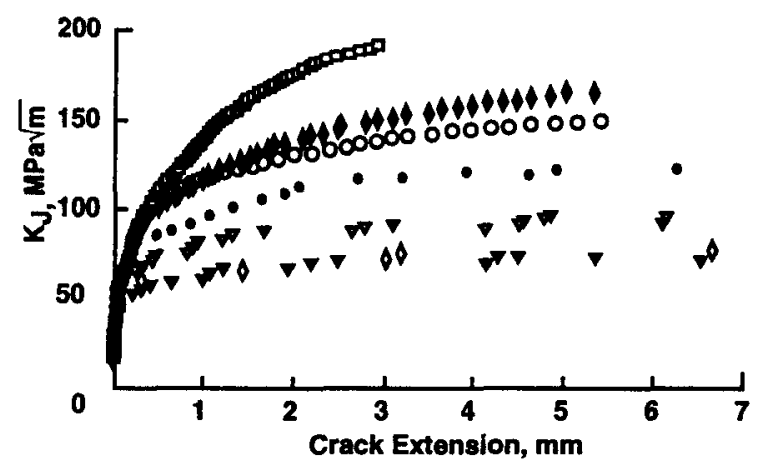

Figure 3. Room temperature crack growth resistance curves for selected titanium sheet.

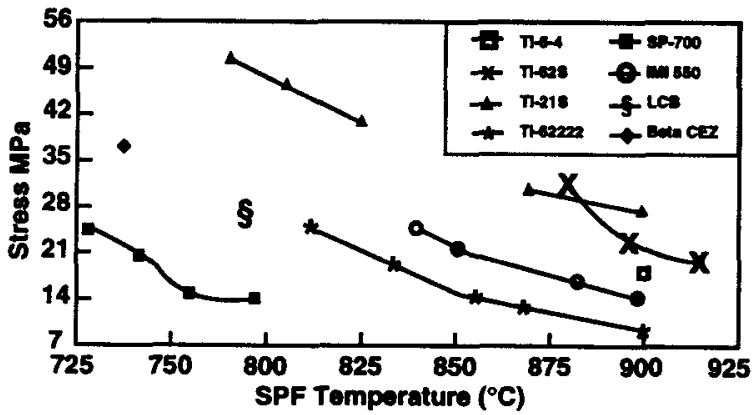

Figure 5. Flow stress vs temperature for selected titanium alloys.

\begin{tabular}{|c|c|c|}
\hline ... & B21S RT data range & $\nabla$ קC: ST $815^{\circ} \mathrm{C} ;$ Age $480^{\circ} \mathrm{C}$ \\
\hline & $\beta 215$ : Direct $a g e 520^{\circ} \mathrm{C} / 11 \mathrm{hr}$ & O pC: ST $815^{\circ} \mathrm{C} ;$ Ago $550^{\circ} \mathrm{C}$ \\
\hline$\Delta$ & $\begin{array}{l}\text { TI-10-2-3: } 740^{\circ} \mathrm{C} / 20 \mathrm{~min} \\
+510^{\circ} \mathrm{Crhhr}\end{array}$ & $\begin{array}{l}\text { प TH6242: RX2 condition } \\
\text { × TL6-2222: ST } 920^{\circ} \mathrm{C} \text {; Ago } 540^{\circ} \mathrm{C}\end{array}$ \\
\hline
\end{tabular}

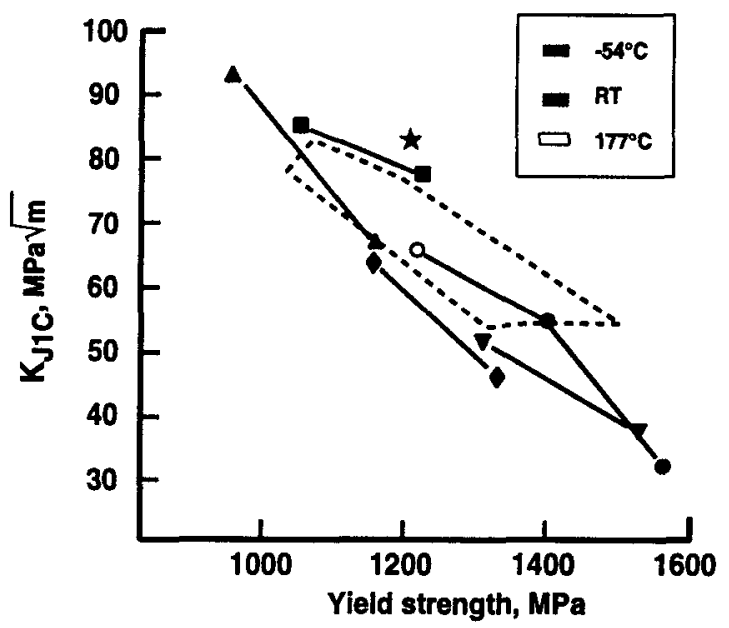

Figure 4. Effect of test temperature on strength and toughness of selected titanium sheet.

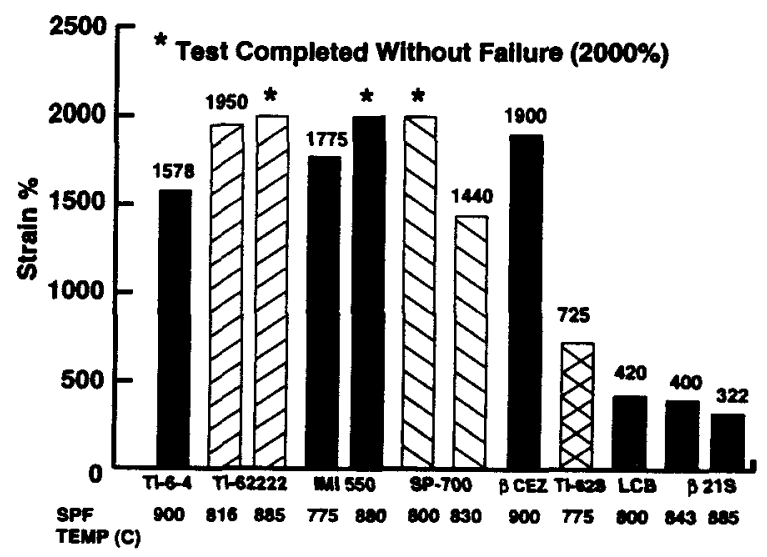

Figure 6. SPF strains for selected titanium alloys. 


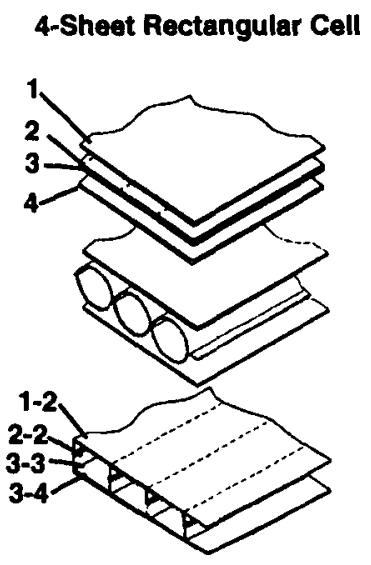

\section{5-Sheet Cross-Web}

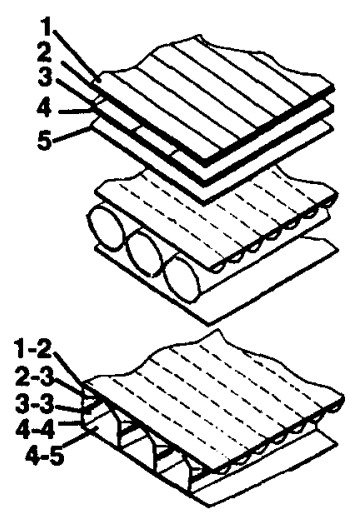

Figure 7. Four \& five sheet RSW/SPF/DB processes.

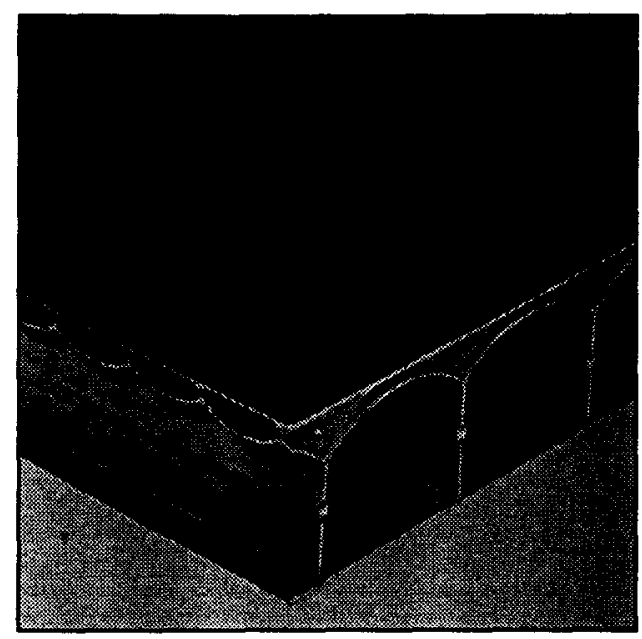

Figure 8. Five sheet cross-webbed structure. Curtesy of Ray Tisler, McDonnell Douglas Aerospace.
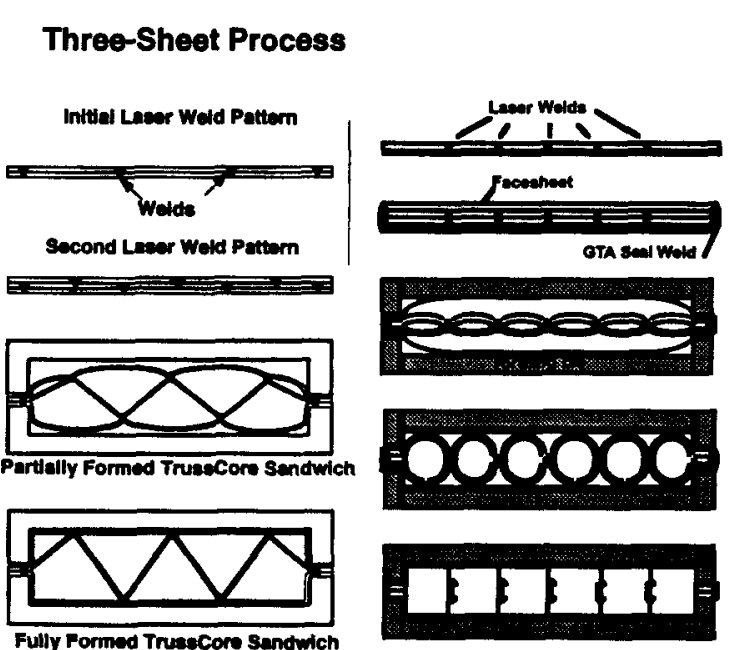

\section{Four-Sheet Process}

- Sheet preparation

- LBW core sheets

- Weld perimeter of core sheets

- Weld on Facesheets \& gas inlet

- Superplastically expand

- Heat treatment

- Alpha case removal
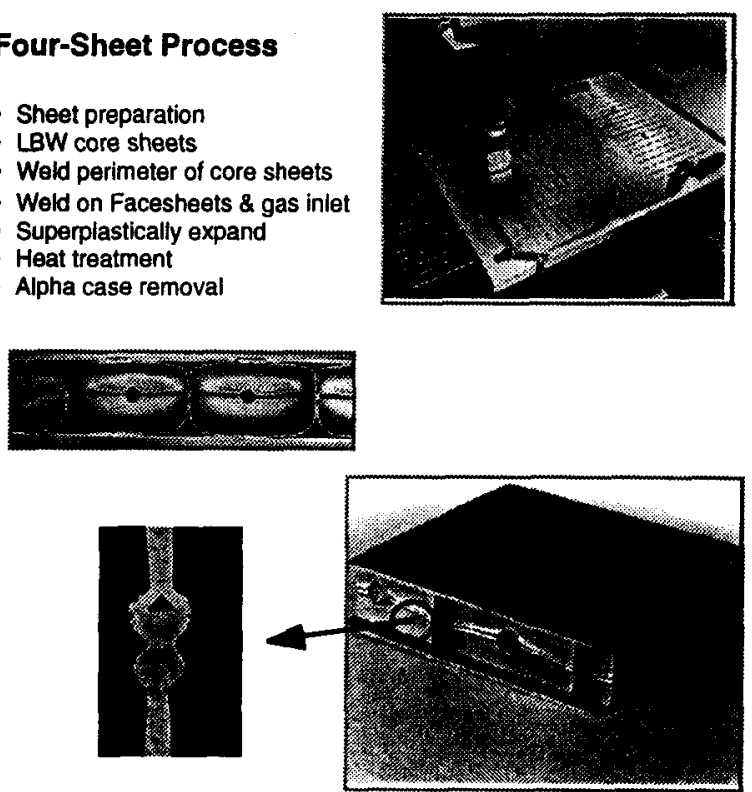

Figure 9. (a) 3 and 4 sheet LW/SPF/DB processes.

(b) Laser welding and 4-sheet structure Curtesy of Jeff Will, Boeing Defense and Space Group 